IOS Press

\title{
On the quality of vocabularies for linked dataset papers published in the Semantic Web journal
}

\author{
Stella Sam ${ }^{\mathrm{a}}$, Pascal Hitzler ${ }^{\mathrm{a},{ }^{*}}$ and Krzysztof Janowicz ${ }^{\mathrm{b}}$ \\ ${ }^{a}$ DaSe Lab, Wright State University, Dayton, OH, USA \\ b STKO Lab, University of California, Santa Barbara, USA
}

\section{Introduction}

Linked Data and knowledge graphs more generally ${ }^{1}$ have been a main driver of research and technology transfer in our field. Linked data serves as a testbed for Semantic Web technologies, as an outreach to application communities interested in data sharing, and contributes more generally to the big data effort by making a large variety of structured data available for all kinds of purposes in various communities [24]. As of December 2017, the LOD Laundromat aggregator [38] alone shows over 38,000,000,000 Linked Data triples which is only a fraction of the data that has been published as Linked Data to date.

Much of Linked Data is generated by researchers as a contribution to the community effort. Due to the importance of this type of data for the advance of research and applications in our field, the Semantic Web journal began in 2012 to solicit papers containing linked dataset descriptions. ${ }^{2}$ The idea behind this was to provide the broader researchers and practitioners commu-

\footnotetext{
*Corresponding author. E-mail: pascal.hitzler@ wright.edu.

${ }^{1}$ There are some notable differences, such as that Linked Data is usually expressed in RDF, perhaps with an OWL ontology, is supposed to be available on the Web, and "linked" with other datasets. Knowledge graphs more broadly speaking would not necessarily conform with any particular syntax, may not be linked to other external sources, and may not even be (easily) accessible on the Web. Nonetheless, many of these differences relate to technologies and not fundamentally different paradigms.

${ }^{2}$ http://www.semantic-web-journal.net/blog/semantic-webjournal-special-call-linked-dataset-descriptions
}

nity with concise summaries of the data, the different tools and endpoints that have been made available to work with the data, worked examples, best practice, and so on, to foster the usage of Linked Data beyond the Semantic Web community. In addition, dataset descriptions allow authors to record their efforts in a citeable paper publication, since this type of credit is still much more important than alternative measures of productivity and impact which take the reuse of other artefacts such as datasets and software into account. The initiative quickly became very popular, ${ }^{3}$ and the first linked dataset papers then appeared in 2013 in the journal [24]. ${ }^{4}$

More recently, the issue of Linked Data quality came into focus [55]. This followed on the heels of application interest and the rise of questions regarding issues such as trustworthiness of content, reliability of resources, or ease of reuse. Indeed in the last few years a significant number of publications have laid out possible quality measures and corresponding algorithms for quality assessment. Truth be told, though, the final verdict is still outstanding regarding the question what dimensions or measures are in fact the most relevant for assessing Linked Data quality.

\footnotetext{
${ }^{3}$ See http://www.semantic-web-journal.net/accepted-datasets for a list of all accepted and/or published SWJ dataset papers.

${ }^{4}$ It should be noted that we have in the meantime increased the bar for such papers, i.e., we have become more selective, in particular regarding tangible evidence for usefulness of the presented datasets. The reason for this is that both community and technology have advanced, and the journal needs to keep up with such developments.
} 
One of the aspects which has so far not received sufficient attention, is the question of relevance of a quality schema or vocabulary for the quality of a linked dataset. If it is indeed relevant, as has been argued [39], then this in turn raises the question how to assess the quality of such schema or vocabulary, in particular since such an evaluation may not be identical to a quality assessment of ontologies independent of a Linked Data context.

To this end, some of us proposed a relatively simple schema - the 5-star LD vocabulary principles, or 5star principles in short - for assessing Linked Data vocabulary quality, in an editorial published in this journal a few years ago [29], as an early contribution to the topic. A majority of the linked dataset papers which the Semantic Web journal has published so far had already been published or submitted, and we did not require adherence to the ideas in that editorial for future submissions, though since the publication of the editorial we have encouraged authors to consider them.

So far, however, there hasn't been any coordinated assessment of the 5-star LD vocabulary principles and how they pan out for real existing datasets. Therefore, in this work, we will look at all linked dataset papers from the perspective of the 5-star vocabulary principles. This serves both as a partial assessment of the quality of linked dataset papers in the Semantic Web journal, and as assessment of the 5-star principles and their applicability in practice. ${ }^{5}$

The paper is structured as follows. Section 2 recalls the 5-star principles and discusses ambiguities and other issues arising when attempting to apply them, as well as explanations on how we resolved these for our present analysis. Section 3 presents the data we have collected, for all linked dataset papers which have so far been published in the Semantic Web journal. Section 4 contains a discussion of the data and what conclusions we can draw from this as we go forward.

\section{The 5-star linked data vocabulary quality categorization and how to apply it}

\subsection{Background and motivation}

In 2010, Tim Berners-Lee published a non-technical schema [5] of awarding anywhere between 0 to 5 stars in rating (Linked) Data depending on how easy it was

\footnotetext{
${ }^{5}$ In a sense, this paper also complements the earlier editorial [26] in which other quality aspects were studied.
}

to discover, use and understand it. However, the 5 Star Linked Data appears to be just a necessary precondition to what is really needed and does not necessarily make the resulting Linked Data more reusable to humans or machines. It does not make any assumptions about the use of vocabularies either. In practice, querying Linked Data that do not refer to a vocabulary is difficult and ascertaining that the results reflect the intended query is impossible. A good vocabulary must restrict potential interpretations of the used classes and roles towards their intended meaning.

In March of 2014, the 5-star LD vocabulary principles [29] were introduced, to encourage data owners, engineers and practitioners to publish and use vocabularies on the Web. Similar to Tim Berners-Lee's stars, which do not directly refer to the quality of the data, this star rating is also not directly concerned with the quality of the vocabularies themselves. Instead, it rates the vocabulary use of a linked dataset following the intuition that data that utilizes well-established, documented, maintained, and interconnected vocabularies is easier to (re)use than Linked Data that may be 5-star data in Berners-Lee's sense but does not utilize such vocabularies.

\subsection{The model}

According to the model, a linked dataset would also be awarded anywhere between 0 to 5 stars as follows:

0 Stars - No web-accessible information on the vocabulary used in the generation of the dataset is available.

1 Star - There exists web-accessible, de-referenceable, human readable information about the vocabulary used.

2 Stars - It is a 1 Star dataset. Also information on the vocabulary used, exists in a machine readable form with explicit axiomatization of it. Ideally RDFS, OWL, RIF or related W3C standards on the Semantic Web stack can be used. This is the level in which automatic reasoning can come into play.

3 Stars - It is a 2 Star dataset. The related vocabulary is linked to other vocabularies. The vocabulary level links must be between classes and properties and not just between individuals. Explicit alignments (e.g. via subClassOf and equivalentClass axioms) are considered better than direct reuse of external vocabularies, though both are acceptable.

4 Stars - It is a 3 Star dataset. Metadata about the related vocabulary such as license model, contact person, last modification date, the ontology used, knowl- 
edge management methodology, etc. used to arrive at the vocabulary is available in a de-referenceable, machine readable format.

5 Stars - It is a 4 Star dataset. Its related vocabulary is linked to by other vocabularies. It is this star that reflects on the external usage and thus usefulness of the vocabulary and thereby ultimately the dataset using it.

We followed this model to manually rate the linked datasets accepted by, described and published in the Semantic Web journal.

\subsection{The rating}

The following steps were followed to rate the datasets:

1. If a vocabulary was developed and used for the generation of the dataset, with the clear presence of a web-accessible, de-referenceable and in human readable form, description of the vocabulary, the 1 st star was awarded to the dataset.

2. If the vocabulary was well-defined with all of the various axioms that comprised it, the 2 nd star was awarded.

3. The 3rd star was awarded, if other standard, wellestablished vocabularies were re-used in a supplemental nature along with (linked to by) this vocabulary, through the use of properties such as subClassOf, subPropertyOf and unionOf. It showed that this vocabulary defines only the required classes and properties that do not already exist in some other standard, well-established vocabulary. If the classes and properties do exist in some other vocabulary, they were re-used by establishing links at the vocabulary level and not at the individual level.

4. The 4th star was awarded, if there was evidence of availability of web-accessible metadata regarding the vocabulary. This ensured that the metadata was available in a standard format such as VoID, VOAF, etc.

5. If it has been clearly shown, that the developed vocabulary has been linked to by other vocabularies to be used in a manner consistent with point 3 above, then the 5th star was awarded.

A total of 49 datasets have been published so far in the Semantic Web journal since 2012 and our analysis, given below, shows that most of them are at least at the 4-star level.

\subsection{Ambiguities \& issues}

The following ambiguities and issues came to light while applying the 5-star principles to the practical reality of the ways vocabularies were used in the generation of datasets.

- For several datasets, the reference links mentioned in the related description paper, do not work and there is no mention on how to find the most recent, updated ones. However, there was ample evidence in the description paper that necessary, web-accessible information regarding the vocabulary used, was present, which resulted in the dataset earning the star rating that it did.

- For the generation of some datasets, no new vocabulary was developed. However, other standard, already well-established vocabularies were reused. This was sufficient for the dataset to earn 4 stars. If the reused vocabulary was, in turn, reused in the generation of a different dataset, the dataset automatically earned 5 stars.

- In some cases, there was no need for the developed vocabulary to be supplemented by other standard, well-established ones, and hence was not linked to other vocabularies, the dataset thus not earning the 3rd star. However, the metadata regarding the vocabulary was present, the dataset thus earning the 4th star without having earned the 3rd star. This was resolved by awarding the dataset 3 stars.

\section{Vocabulary quality for SWJ linked datasets}

Table 1 presents the data from our analysis for the 49 papers and the star rating of vocabulary use.

\section{Discussion}

At this stage, our collected data is mainly descriptive. The editorial introducing the 5-star vocabulary categories was about the presentation of a position on the importance of Linked Data vocabularies and a anticipation on what aspects may be indicative of quality while at the same time providing simple guidelines and rules. As such, it was meant as a starting point for discussions, rather than as an end in itself. And as we write this new editorial, the discussion regarding the question what aspects of linked datasets are actually important for quality, is still ongoing. 
Table 1

Linked datasets analysis

\begin{tabular}{|c|c|c|c|c|c|c|}
\hline Linked dataset & $\begin{array}{l}\text { Published } \\
\text { date }\end{array}$ & Base vocabulary & Linked to vocabulary & Linked from vocabulary & $\begin{array}{c}\text { Star } \\
\text { rating }\end{array}$ & Comments \\
\hline $\begin{array}{l}\text { A Curated and Evolving } \\
\text { Linguistic Linked Dataset [6] }\end{array}$ & 2012 & ASit Vocabulary & Geonames Ontology & & 4 & \\
\hline $\begin{array}{l}\text { Amsterdam Museum Linked } \\
\text { Open Data [14] }\end{array}$ & 2012 & $\begin{array}{l}\text { Extends EDM, OAI-ORE } \\
\text { model }\end{array}$ & $\begin{array}{l}\text { EDM, OAI-ORE, } \\
\text { Thesaurus to AATNed, } \\
\text { DBPedia, ULAN,Thesaurus } \\
\text { to GeoNames, RDA }\end{array}$ & & 4 & \\
\hline $\begin{array}{l}\text { BioPortal as a Dataset of } \\
\text { Linked Biomedical } \\
\text { Ontologies and } \\
\text { Terminologies in RDF [41] }\end{array}$ & 2012 & $\begin{array}{l}\text { BioPortal Metadata } \\
\text { Ontology extends OMV }\end{array}$ & SKOS, OMV & & 4 & $\begin{array}{l}\text { BioPortal Metadata } \\
\text { Ontology extends OMV - } \\
\text { Ontology Metadata } \\
\text { Vocabulary - to track a set } \\
\text { of metadata related to } \\
\text { each ontology in the } \\
\text { system }\end{array}$ \\
\hline $\begin{array}{l}\text { datos.bne.es: a Library } \\
\text { Linked Data Dataset [49] }\end{array}$ & 2012 & $\begin{array}{l}\text { Modelled using well } \\
\text { established vocabularies: } \\
\text { RDA, Dublin Core } \\
\text { Metadata Element Set, } \\
\text { BioPortal as a Dataset of } \\
\text { Linked Biomedical } \\
\text { Ontologies and } \\
\text { Terminologies in RDF \& } \\
\text { IFLA approved ontologies: } \\
\text { FRBR, FRAD, FRSAD, } \\
\text { ISBD }\end{array}$ & $\begin{array}{l}\text { VIAF, GND18, DBpedia, } \\
\text { Libris, SUDOC, Lexvo }\end{array}$ & & 4 & \\
\hline $\begin{array}{l}\text { Europeana Linked Open } \\
\text { Data - data.europeana.eu } \\
\text { [27] }\end{array}$ & 2012 & EDM & $\begin{array}{l}\text { Aligned to: Dublin Core, } \\
\text { SKOS, OAI-ORE, } \\
\text { CIDOC-CRM, GEMET } \\
\text { Thesaurus, Semium } \\
\text { ontology }\end{array}$ & $\begin{array}{l}\text { The Amsterdam Museum } \\
\text { Prototype uses EDM }\end{array}$ & 5 & $\begin{array}{l}\text { Europeana Semantic } \\
\text { Elements (ESE) XML } \\
\text { Schema to EDM is done } \\
\text { manually }\end{array}$ \\
\hline $\begin{array}{l}\text { Facebook Linked Data via } \\
\text { the Graph API [51] }\end{array}$ & 2012 & Extends RDFS, OWL & RDFS, OWL & & 4 & \\
\hline $\begin{array}{l}\text { Fiction Literature as Linked } \\
\text { Open Data - the BookSampo } \\
\text { Dataset [33] }\end{array}$ & 2012 & $\begin{array}{l}\text { Aligned with the ontologies } \\
\text { of CultureSampo, KOKO a } \\
\text { lightweight ontology } \\
\text { comprising } 14 \text { domain } \\
\text { ontologies joined under } \\
\text { Finnish National Upper } \\
\text { Ontology YSO }\end{array}$ & $\begin{array}{l}\text { KOKO lightweight } \\
\text { ontology comprising } 14 \\
\text { domain ontologies joined } \\
\text { under Finnish National } \\
\text { Upper Ontology YSO, } \\
\text { LEXVO language ontology }\end{array}$ & & 4 & $\begin{array}{l}\text { Metadata was transformed } \\
\text { to RDF format from } \\
\text { legacy libraries and then } \\
\text { manually enriched by } \\
\text { dozens of Finnish } \\
\text { Librarians using SAHA a } \\
\text { metadata editor }\end{array}$ \\
\hline
\end{tabular}


Table 1

(Continued)

\begin{tabular}{|c|c|c|c|c|c|c|}
\hline Linked dataset & $\begin{array}{c}\text { Published } \\
\text { date }\end{array}$ & Base vocabulary & Linked to vocabulary & Linked from vocabulary & $\begin{array}{c}\text { Star } \\
\text { rating }\end{array}$ & Comments \\
\hline $\begin{array}{l}\text { Linked Brazilian Amazon } \\
\text { Rainforest Data [30] }\end{array}$ & 2012 & $\begin{array}{l}\text { OLA, TISC as well as other } \\
\text { well-established } \\
\text { vocabularies }\end{array}$ & TISC Vocabulary & & 4 & \\
\hline $\begin{array}{l}\text { Linked data for Potential } \\
\text { Algal Biomass Production } \\
\text { [45] }\end{array}$ & 2012 & $\begin{array}{l}\text { WGS84 ontology, spatial } \\
\text { relations ontology, the } \\
\text { Geonames ontology and the } \\
\text { NeoGeo ontology, } \\
\text { extension of Neo Geo } \\
\text { Geometry Ontology, QUDT } \\
\text { Ontology, NUTS } \\
\text { Vocabulary }\end{array}$ & $\begin{array}{l}\text { All vocabularies used are } \\
\text { established ones. The } \\
\text { LEAPS datasets are } \\
\text { interlinked with each other } \\
\text { through their respective } \\
\text { vocabularies }\end{array}$ & & 4 & \\
\hline $\begin{array}{l}\text { Linked Nomenclature of } \\
\text { Territorial Units for Statistics } \\
\text { [12] }\end{array}$ & 2012 & $\begin{array}{l}\text { Extends OS (Ordinance } \\
\text { Survey) Ontology, OWL } \\
\text { Time Ontology }\end{array}$ & $\begin{array}{l}\text { OS Ontology, OWL Time } \\
\text { Ontology }\end{array}$ & & 4 & \\
\hline $\begin{array}{l}\text { The AGROVOC Linked } \\
\text { Dataset [9] }\end{array}$ & 2012 & AGROVOC (SKOS-XL) & $\begin{array}{l}\text { Linked to } 13 \text { Vocabularies, } \\
\text { Thesauri \& Ontologies: The } \\
\text { Library of Congress } \\
\text { Subject Headings (LCSH), } \\
\text { RAMEAU Répertoire } \\
\text { d'autoritématière } \\
\text { encyclopedique et } \\
\text { alphabetique unifie, } \\
\text { EUROVOC, DBpedia, } \\
\text { experimental Linked Data } \\
\text { version of the Dewey } \\
\text { Decimal Classification, } \\
\text { NAL Thesaurus for } \\
\text { agriculture, GEMETfor } \\
\text { environment, STW for } \\
\text { Economics, TheSoz for } \\
\text { social science, both } \\
\text { GeoNames and FAO } \\
\text { Geopolitical Ontology } \\
\text { cover countries and } \\
\text { political regions, ASFA17 } \\
\text { covers aquatic science \& } \\
\text { Biotechnology glossary } \\
\text { covers biotechnology }\end{array}$ & $\begin{array}{l}\text { Aligned to the following } \\
\text { Vocabularies: ASFA, } \\
\text { Biotechnology Glossary } \\
\text { (FAO), Chinese Agriculture } \\
\text { Thesaurus (CAT), } \\
\text { DBPedia, Dewey Decimal } \\
\text { Classification (DDC), } \\
\text { EUROVOC, GEMET, } \\
\text { GeoNames, Geopolitical } \\
\text { Ontology, Library of } \\
\text { Congress Subject Headings } \\
\text { (LCSH), NAL Thesaurus, } \\
\text { RAMEAU Répertoire } \\
\text { d'autoritématière } \\
\text { encyclopedique et } \\
\text { alphabetique unifie, STW - } \\
\text { Thesaurus for Economics, } \\
\text { TheSoz - Thesaurus for } \\
\text { Social Sciences, SWD } \\
\text { (Schlagwortnormdatei), } \\
\text { EARTh }\end{array}$ & 5 & $\begin{array}{l}\text { SKOS-XL (SKOS } \\
\text { Extension for Labels) has } \\
\text { been used for the } \\
\text { EUROVOC Thesaurus }\end{array}$ \\
\hline
\end{tabular}


Table 1

(Continued)

\begin{tabular}{|c|c|c|c|c|c|c|}
\hline Linked dataset & $\begin{array}{l}\text { Published } \\
\text { date }\end{array}$ & Base vocabulary & Linked to vocabulary & Linked from vocabulary & $\begin{array}{c}\text { Star } \\
\text { rating }\end{array}$ & Comments \\
\hline $\begin{array}{l}\text { TheSoz: A SKOS } \\
\text { Representation of the } \\
\text { Thesaurus for the Social } \\
\text { Sciences [53] }\end{array}$ & 2012 & SKOS, SKOS-XL & $\begin{array}{l}\text { Creative Commons, } \\
\text { Provenance }\end{array}$ & Void & 5 & $\begin{array}{l}\text { SKOS-XL (SKOS } \\
\text { Extension for Labels) has } \\
\text { been used for the } \\
\text { EUROVOC Thesaurus }\end{array}$ \\
\hline $\begin{array}{l}\text { TourMISLOD: a Tourism } \\
\text { Linked Data Set [40] }\end{array}$ & 2012 & $\begin{array}{l}\text { TOURMIS ontology - } \\
\text { Schema based on } \\
\text { dbpedia.com, xsd \& text }\end{array}$ & DBPedia,GeoNames & & 4 & $\begin{array}{l}\text { Closed license because of } \\
\text { the heterogeneous nature } \\
\text { of its contributors }\end{array}$ \\
\hline $\begin{array}{l}\text { Transforming Meteorological } \\
\text { Data into Linked Data [3] }\end{array}$ & 2012 & $\begin{array}{l}\text { AEMET ontology (an } \\
\text { extension of W3C SSN - } \\
\text { Semantic Sensor Network } \\
\text { ontology) comprises: } \\
\text { 1. Measurement Ontology - } \\
\text { Mainly reuse from SSN. } \\
\text { 2. Location Ontology - } \\
\text { Mainly reuse from } \\
\text { wgs84_pos ontology; also } \\
\text { reuse from GeoBuddies } \\
\text { ontology. 3. Time } \\
\text { Ontology - Reuse from } \\
\text { OWL Time ontology. } \\
\text { 4. Sensor Ontology - } \\
\text { Extension of SSN ontology }\end{array}$ & $\begin{array}{l}\text { GeoLinkedDataset, } \\
\text { DBPedia }\end{array}$ & & 4 & \\
\hline $\begin{array}{l}\text { Translational research } \\
\text { combining orthologous genes } \\
\text { and human diseases with the } \\
\text { OGOLOD dataset [35] }\end{array}$ & 2012 & OGO Ontology & $\begin{array}{l}\text { GO, ECO, NCBI taxonomy, } \\
\text { RO, HPO }\end{array}$ & & 4 & \\
\hline $\begin{array}{l}\text { Converting neXtProt into } \\
\text { Linked Data and } \\
\text { nanopublications [11] }\end{array}$ & 2013 & PTM nanopublication & $\begin{array}{l}\text { BioPAX ontology, } \\
\text { Semantic science Integrated } \\
\text { Ontology (SIO), Weighted } \\
\text { Interests Vocabulary }\end{array}$ & & 4 & $\begin{array}{l}\text { Many of the reference } \\
\text { links provided do not } \\
\text { work }\end{array}$ \\
\hline $\begin{array}{l}\text { eagle-i: biomedical research } \\
\text { resource datasets [46] }\end{array}$ & 2013 & $\begin{array}{l}\text { ERO (Eagle-I Resource } \\
\text { Ontology) }\end{array}$ & $\begin{array}{l}\text { Gene Ontology, DBPedia } \\
\text { through OWL sameAs }\end{array}$ & $\begin{array}{l}\text { Has been generalized and } \\
\text { re-used in Reagent } \\
\text { Ontology (ReO) and the } \\
\text { Agent, Resource \& Grant } \\
\text { ontology }\end{array}$ & 5 & \\
\hline
\end{tabular}


Table 1

(Continued)

\begin{tabular}{|c|c|c|c|c|c|c|}
\hline Linked dataset & $\begin{array}{l}\text { Published } \\
\text { date }\end{array}$ & Base vocabulary & Linked to vocabulary & Linked from vocabulary & $\begin{array}{c}\text { Star } \\
\text { rating }\end{array}$ & Comments \\
\hline $\begin{array}{l}\text { EARTh: an Environmental } \\
\text { Application Reference } \\
\text { Thesaurus in the Linked } \\
\text { Open Data Cloud [1] }\end{array}$ & 2013 & SKOS - extends GEMET & $\begin{array}{l}\text { Interlinks to: AGROVOC, } \\
\text { EUROVOC, } \\
\text { DBPEDIA,UMTHES } \\
\text { Thesauri, each of them } \\
\text { having their own } \\
\text { vocabulary }\end{array}$ & $\begin{array}{l}\text { NatureSDIplus \& } \\
\text { eENVplus employs EARTh } \\
\text { as a backbone thesaurus }\end{array}$ & 5 & \\
\hline $\begin{array}{l}\text { Geospatial Dataset Curation } \\
\text { through a Location-based } \\
\text { Game [10] }\end{array}$ & 2013 & $\begin{array}{l}\text { Urbanopoly Ontology, } \\
\text { Human Computation } \\
\text { Ontology. } \\
\text { Also linked to ontologies: } \\
\text { OpenStreetMap, } \\
\text { LinkedGeoData }\end{array}$ & $\begin{array}{l}\text { Human Computation } \\
\text { Ontology extends the } \\
\text { PROV-O ontology }\end{array}$ & & 4 & $\begin{array}{l}\text { Any Human Computation } \\
\text { effort can be modelled } \\
\text { using the Human } \\
\text { Computation ontology }\end{array}$ \\
\hline $\begin{array}{l}\text { Increasing the Financial } \\
\text { Transparency of European } \\
\text { Commission Project Funding } \\
\text { [34] }\end{array}$ & 2013 & FTS Ontology & $\begin{array}{l}\text { Sub-Property links to } \\
\text { DBPedia (close connection } \\
\text { to the DBPedia Vocabulary) }\end{array}$ & & 4 & \\
\hline $\begin{array}{l}\text { Lexvo.org: } \\
\text { Language-Related } \\
\text { Information for the } \\
\text { Linguistic Linked Data } \\
\text { Cloud [15] }\end{array}$ & 2013 & Lexvo Ontology & $\begin{array}{l}\text { GEneral Multilingual } \\
\text { Environmental Thesaurus } \\
\text { (GEMET), the United } \\
\text { Nations FAO AGROVOC } \\
\text { thesaurus, the US National } \\
\text { Agricultural Library } \\
\text { Thesaurus, EuroVoc, } \\
\text { RAMEAU subject } \\
\text { headings. } \\
\text { Links to upper ontologies } \\
\text { such as OpenCyc are } \\
\text { provided as well }\end{array}$ & & 4 & \\
\hline $\begin{array}{l}\text { Linked European Television } \\
\text { Heritage [44] }\end{array}$ & 2013 & $\begin{array}{l}\text { EBU Core ontology - RDF } \\
\text { representation of the EBU } \\
\text { Class Conceptual Data } \\
\text { Model (CCDM), MetaData } \\
\text { Model: EU Screen } \\
\text { Harvesting Schema }\end{array}$ & $\begin{array}{l}\text { W3C Media Annotation } \\
\text { Ontology (W3C MAWG), } \\
\text { EDM for provenance } \\
\text { related information }\end{array}$ & & 4 & \\
\hline Linked SDMX Data [8] & 2013 & $\begin{array}{l}\text { RDF Data Cube vocabulary, } \\
\text { SKOS, XKOS, PROV-O }\end{array}$ & $\begin{array}{l}\text { RDF Data Cube vocabulary, } \\
\text { SKOS, XKOS, PROV-O }\end{array}$ & & 4 & \\
\hline
\end{tabular}


Table 1

(Continued)

\begin{tabular}{|c|c|c|c|c|c|c|}
\hline Linked dataset & $\begin{array}{l}\text { Published } \\
\text { date }\end{array}$ & Base vocabulary & Linked to vocabulary & Linked from vocabulary & $\begin{array}{c}\text { Star } \\
\text { rating }\end{array}$ & Comments \\
\hline $\begin{array}{l}\text { Migrating Bibliographic } \\
\text { Datasets to the Semantic } \\
\text { Web: the AGRIS case [2] }\end{array}$ & 2013 & BIBO, FOAF, Dublin Core & $\begin{array}{l}\text { AGROVOC, RDF/SKOS - } \\
\text { XL Concept Scheme } \\
\text { aligned to } 16 \text { other } \\
\text { vocabularies:ASFA, FAO } \\
\text { Biotechnology Glossary, } \\
\text { Chinese Agriculture } \\
\text { Thesaurus (CAT), EARTh, } \\
\text { Eurovoc, GEMET, Library } \\
\text { of Congress Subject } \\
\text { Headings (LCSH), NAL, } \\
\text { RAMEAU, STW - } \\
\text { Thesaurus for Economics, } \\
\text { TheSoz - Thesaurus for the } \\
\text { Social Sciences, Geopolical } \\
\text { Ontology, Dewey Decimal } \\
\text { Classification (DDC), } \\
\text { DBPedia, SWD } \\
\text { (Schlagwortnormdatei), } \\
\text { GeoNames }\end{array}$ & & 4 & \\
\hline $\begin{array}{l}\text { Public spending as LOD: the } \\
\text { case of Greece [47] }\end{array}$ & 2013 & PS Ontology & DBPedia, Geonames & & 4 & \\
\hline $\begin{array}{l}\text { Publishing and Interlinking } \\
\text { the Global Health } \\
\text { Observatory Dataset [54] }\end{array}$ & 2013 & RDF Data Cube & & & 3 & $\begin{array}{l}\text { Vocabulary has meta data } \\
\text { but is not linked to any } \\
\text { other vocabulary }\end{array}$ \\
\hline $\begin{array}{l}\text { Semantic Quran a } \\
\text { Multilingual Resource for } \\
\text { Natural-Language Processing } \\
\text { [43] }\end{array}$ & 2013 & $\begin{array}{l}\text { The Quran Schema } \\
\text { Vocabulary (qvoc) }\end{array}$ & $\begin{array}{l}\text { Extends GOLD linguistic } \\
\text { ontology \& OLIA Arabic } \\
\text { linguistic ontology }\end{array}$ & & 4 & \\
\hline $\begin{array}{l}\text { Converting the PAROLE } \\
\text { SIMPLE CLIPS Lexicon into } \\
\text { RDF with lemon [23] }\end{array}$ & 2014 & $\begin{array}{l}\text { LEMON,SIMPLE-OWL } \\
\text { ontology }\end{array}$ & & & 5 & $\begin{array}{l}\text { Vocabulary has meta data } \\
\text { but is not linked to any } \\
\text { other vocabulary. Base } \\
\text { Vocabulary used makes it } \\
\text { a 5-star dataset }\end{array}$ \\
\hline $\begin{array}{l}\text { Countering language attrition } \\
\text { with PanLex and the Web of } \\
\text { Data [52] }\end{array}$ & 2014 & PanLex RDF vocabulary & $\begin{array}{l}\text { LexVo, Dbpedia, GOLD, } \\
\text { LEMON }\end{array}$ & & 4 & \\
\hline
\end{tabular}


Table 1

(Continued)

\begin{tabular}{|c|c|c|c|c|c|c|}
\hline Linked dataset & $\begin{array}{l}\text { Published } \\
\text { date }\end{array}$ & Base vocabulary & Linked to vocabulary & Linked from vocabulary & $\begin{array}{c}\text { Star } \\
\text { rating }\end{array}$ & Comments \\
\hline $\begin{array}{l}\text { DBnary: Wiktionary as a } \\
\text { Lemon-Based Multilingual } \\
\text { Lexical Resource in RDF } \\
\text { [42] }\end{array}$ & 2014 & Dbnary Ontology & $\begin{array}{l}\text { Based on the LEMON } \\
\text { ontology }\end{array}$ & & 4 & $\begin{array}{l}\text { Classes \& properties are } \\
\text { added on to LEMON } \\
\text { when required }\end{array}$ \\
\hline $\begin{array}{l}\text { EventMedia: a LOD Dataset } \\
\text { of Events Illustrated with } \\
\text { Media [31] }\end{array}$ & 2014 & $\begin{array}{l}\text { LODE ontology, Media } \\
\text { Resources ontology }\end{array}$ & $\begin{array}{l}\text { W3C Ontologies for Media } \\
\text { Resources, SIOC,VCard }\end{array}$ & & 4 & \\
\hline $\begin{array}{l}\text { lemonUby - a large, } \\
\text { interlinked, syntactically-rich } \\
\text { lexical resource for } \\
\text { ontologies [19] }\end{array}$ & 2014 & $\begin{array}{l}\text { UBY-LMF is mapped to } \\
\text { Lemon called lemonUby } \\
\text { model, Ontology lexica, } \\
\text { OWL/DL ontology, } \\
\text { UBYCat (to link to ISOCat) }\end{array}$ & $\begin{array}{l}\text { UbyCat linked to Ontologies } \\
\text { of Linguistic Annotations } \\
\text { (Olia) }\end{array}$ & & 4 & $\begin{array}{l}\text { Through Olia, it is } \\
\text { possible to interlink } \\
\text { lemonUby with other } \\
\text { LLOD linked to either } \\
\text { Olia or any of the } \\
\text { terminology repositories } \\
\text { like GOLD \& ISOCat }\end{array}$ \\
\hline $\begin{array}{l}\text { LinkedSpending: } \\
\text { OpenSpending becomes } \\
\text { Linked Open Data [25] }\end{array}$ & 2014 & $\begin{array}{l}\text { RDF Data Cube } \\
\text { Vocabulary, SDMX, XSD, } \\
\text { DBPedia, LinkedGeoData }\end{array}$ & $\begin{array}{l}\text { DBPedia, Linked GeoData } \\
\text { SDMX }\end{array}$ & & 4 & $\begin{array}{l}\text { The cube models } \\
\text { underlying both Linked } \\
\text { GeoData SDMX as well } \\
\text { as RDF Data Cube } \\
\text { vocabularies are } \\
\text { compatible }\end{array}$ \\
\hline $\begin{array}{l}\text { PAROLE/SIMPLE 'Lemon' } \\
\text { ontology and lexicons [50] }\end{array}$ & 2014 & PAROLE/SIMPLE Model & $\begin{array}{l}\text { WordNet, lemonUby, } \\
\text { DBpedia }\end{array}$ & & 4 & $\begin{array}{l}\text { Mapped onto LEMON } \\
\text { Model }\end{array}$ \\
\hline $\begin{array}{l}\text { CEDAR: The Dutch } \\
\text { Historical Censuses as } \\
\text { Linked Open Data [36] }\end{array}$ & 2015 & CEDAR & $\begin{array}{l}\text { RDF Data Cube, Open } \\
\text { Annotation, SDMX, PROV } \\
\text { vocabularies }\end{array}$ & & 4 & \\
\hline $\begin{array}{l}\text { Facilitating Scientometrics in } \\
\text { Learning Analytics and } \\
\text { Educational Data Mining - } \\
\text { the LAK Dataset [16] }\end{array}$ & 2015 & $\begin{array}{l}\text { BIBO, FOAF, SWRC, } \\
\text { Schema.org, SWC }\end{array}$ & $\begin{array}{l}\text { DBPedia is used as base } \\
\text { vocabulary }\end{array}$ & & 4 & \\
\hline $\begin{array}{l}\text { Publishing DisGeNET as } \\
\text { Nanopublications [37] }\end{array}$ & 2015 & $\begin{array}{l}\text { DisGeNET } \\
\text { nanopublications }\end{array}$ & $\begin{array}{l}\text { SIO, NcIt, PROV-O, } \\
\text { Provenance, Authoring and } \\
\text { Versioning (PAV) vocabulary, } \\
\text { Provenance Vocabulary Core } \\
\text { Ontology Specification } \\
\text { (PRV), Weighted Interests } \\
\text { vocabulary (WI), Evidence } \\
\text { Codes Ontology (ECO) }\end{array}$ & & 4 & \\
\hline
\end{tabular}


(Continued)

\begin{tabular}{|c|c|c|c|c|c|c|}
\hline Linked dataset & $\begin{array}{l}\text { Published } \\
\text { date }\end{array}$ & Base vocabulary & Linked to vocabulary & Linked from vocabulary & $\begin{array}{c}\text { Star } \\
\text { rating }\end{array}$ & Comments \\
\hline $\begin{array}{l}\text { The Open University Linked } \\
\text { Data - data.open.ac.uk [13] }\end{array}$ & 2015 & $\begin{array}{l}\text { FOAF, SKOS, SIOC, OWL, } \\
\text { Dublin Core, Bibo, XCRI }\end{array}$ & $\begin{array}{l}\text { FOAF, SKOS, SIOC, OWL, } \\
\text { Dublin Core, Bibo, XCRI }\end{array}$ & & 4 & $\begin{array}{l}\text { Reuses vocabularies to the } \\
\text { most extent possible } \\
\text { (57 vocabularies) }\end{array}$ \\
\hline $\begin{array}{l}\text { A Linked Data Wrapper for } \\
\text { CrunchBase [21] }\end{array}$ & 2016 & CrunchBase Schema & $\begin{array}{l}\text { Schema description in OWL } \\
\& \text { vocabulary description in } \\
\text { VoiD }\end{array}$ & & 4 & $\begin{array}{l}\text { Many of the reference } \\
\text { links provided do not } \\
\text { work }\end{array}$ \\
\hline $\begin{array}{l}\text { DM2E: A Linked Data } \\
\text { Source of Digitised } \\
\text { Manuscripts for the Digital } \\
\text { Humanities [4] }\end{array}$ & 2016 & $\begin{array}{l}\text { DM2E model based on } \\
\text { OAI-ORE, Dublin Core and } \\
\text { SKOS }\end{array}$ & $\begin{array}{l}\text { Reused vocabularies: } \\
\text { BIBFRAME, BIBO, } \\
\text { CIDOC-CRM, FABIO, PRO, } \\
\text { rdaGr2, VIVO }\end{array}$ & & 4 & \\
\hline $\begin{array}{l}\text { JRC-Names: Multilingual } \\
\text { Entity Name variants and } \\
\text { titles as Linked Data [20] }\end{array}$ & 2016 & JRC-Model & $\begin{array}{l}\text { Reuses: Lemon, Lexinfo, } \\
\text { OLiA, JRC links to } \\
\text { vocabulary of NYT - New } \\
\text { York Times (but loosely) }\end{array}$ & & 4 & \\
\hline $\begin{array}{l}\text { Linked Web APIs Dataset: } \\
\text { Web APIs meet Linked Data } \\
{[18]}\end{array}$ & 2016 & $\begin{array}{l}\text { Linked Web APIs } \\
\text { Ontology, LWAPIS } \\
\text { (RDF/OWL based) }\end{array}$ & $\begin{array}{l}\text { Integrates: FOAF, Dublin } \\
\text { Core, WSMO-Lite, MSM, } \\
\text { PROV Ontology }\end{array}$ & & 4 & \\
\hline $\begin{array}{l}\text { Meta-Data for a lot of LOD } \\
\text { [38] }\end{array}$ & 2016 & $\begin{array}{l}\text { LOD Laundromat based on: } \\
\text { Bio2RDF, PROV, Dce, Dct, } \\
\text { VANN, VOID, VOID-ext }\end{array}$ & $\begin{array}{l}\text { PROV-O, HTTP Vocabulary, } \\
\text { Error Ontology }\end{array}$ & & 4 & \\
\hline $\begin{array}{l}\text { Migration of a library } \\
\text { catalogue into RDA linked } \\
\text { open data [7] }\end{array}$ & 2016 & $\begin{array}{l}\text { RDA based on models: } \\
\text { FRBR, FRAD, FRSAD }\end{array}$ & $\begin{array}{l}\text { OWL Time ontology, FOAF, } \\
\text { Dublin Core, Library of } \\
\text { Congress MetaData } \\
\text { Authority Description } \\
\text { Schema, DBPedia-OWL } \\
\text { ontology }\end{array}$ & & 4 & \\
\hline $\begin{array}{l}\text { The ACORN-SAT Linked } \\
\text { Climate Dataset [32] }\end{array}$ & 2016 & $\begin{array}{l}\text { W3C RDF Data Cube } \\
\text { Vocabulary, W3C Semantic } \\
\text { Sensor Network ontology, } \\
\text { acorn-sat observation } \\
\text { ontology, acorn-series time } \\
\text { series ontology, climate } \\
\text { ontology, raindist rainfall } \\
\text { district ontology }\end{array}$ & $\begin{array}{l}\text { Vocabulary of Interlinked } \\
\text { Datasets: RDF Data Cube } \\
\text { Vocabulary, Simple } \\
\text { Knowledge Organization } \\
\text { System, Semantic Sensor } \\
\text { Network ontology, } \\
\text { GeoNames ontology (version } \\
\text { 3.1), Basic Geo (WGS84 } \\
\text { lat/long) vocabulary, Time } \\
\text { Ontology in OWL, } \\
\text { DOLCE+DnS Ultralite }\end{array}$ & $\begin{array}{l}\text { External datasets already } \\
\text { SSN Ontology based link } \\
\text { to it }\end{array}$ & 5 & \\
\hline
\end{tabular}


Table 1

(Continued)

\begin{tabular}{|c|c|c|c|c|c|c|}
\hline Linked dataset & $\begin{array}{l}\text { Published } \\
\text { date }\end{array}$ & Base vocabulary & Linked to vocabulary & Linked from vocabulary & $\begin{array}{c}\text { Star } \\
\text { rating }\end{array}$ & Comments \\
\hline $\begin{array}{l}\text { The Apertium Bilingual } \\
\text { Dictionaries on the Web of } \\
\text { Data [22] }\end{array}$ & 2016 & $\begin{array}{l}\text { Lemon, Lexinfo, Lemon } \\
\text { translation module }\end{array}$ & Lemon, lexinfo & $\begin{array}{l}\text { Lemon along with lemon } \\
\text { translation module serves } \\
\text { as the basis for the } \\
\text { lemon-ontolex model }\end{array}$ & 5 & \\
\hline $\begin{array}{l}\text { The debates of the European } \\
\text { Parliament as Linked Open } \\
\text { Data [48] }\end{array}$ & 2016 & Vocabulary for LinkedEP & $\begin{array}{l}\text { Links to } 4 \text { external sources } \\
\text { (2 on politicians' } \\
\text { backgrounds, } \\
1 \text { geographical database } \\
\text { and } 1 \text { on topic taxonomy): } \\
\text { 1) LinkedEP to DBPedia - } \\
\text { RDF, YAGO Ontology } \\
\text { 2) LinkedEP MEP to the } \\
\text { Italian Parliament - } \\
\text { dati.camera.it (dublin core, } \\
\text { foaf, BIO vocabularies) } \\
\text { 3) LinkedEP Countries to } \\
\text { GeoNames - GeoNames } \\
\text { Ontology 4) LinkedEP } \\
\text { Agenda Items to Eurovoc } \\
\text { Thesaurus }\end{array}$ & & 4 & \\
\hline $\begin{array}{l}\text { The Rijksmuseum Collection } \\
\text { as Linked Data [17] }\end{array}$ & 2016 & $\begin{array}{l}\text { EDM \& RDA (Resource } \\
\text { Description and Access } \\
\text { Vocabulary), AAT (Arts }\end{array}$ & $\begin{array}{l}\text { Architecture Thesaurus), } \\
\text { IconClass, STCN (Short } \\
\text { Title catalogue } \\
\text { Netherlands) are used to } \\
\text { extend it }\end{array}$ & & 4 & $\begin{array}{l}\text { Though the base model is } \\
\text { EDM, others access it } \\
\text { only through APIs }\end{array}$ \\
\hline $\begin{array}{l}\text { Wikidata through the Eyes of } \\
\text { DBpedia [28] }\end{array}$ & 2016 & $\begin{array}{l}\text { Own data model to better } \\
\text { capture provenance } \\
\text { information (not RDF } \\
\text { based but using this as base, } \\
\text { different RDF serializations } \\
\text { are possible). DBPedia } \\
\text { ontology and RDF } \\
\text { reification }\end{array}$ & $\begin{array}{l}\text { OWL punning used to } \\
\text { define owl:equivalentClass } \\
\text { to links between DBPedia } \\
\text { classes and related } \\
\text { Wikidata items }\end{array}$ & & 3 & $\begin{array}{l}\text { Part of the DBPedia } \\
\text { publishing work-flow }\end{array}$ \\
\hline
\end{tabular}


Likewise, the point of publishing our analysis herein lies in stimulating further discussion.

Based on our analysis, a case may be made for interchanging the orders of the third and fourth stars of the 5 -star model. The first 3 stars would then be a reflection on the completeness of information on the vocabulary itself, while the next 2 stars would be a reflection on the vocabulary's interactions with other vocabularies. Furthermore, this would avoid the occurrence of a dataset getting a fourth star without getting the third star, in some cases. Of course, if the metadata of the vocabulary were not defined, then we could still see this happening.

Our assessment shows that all linked datasets with corresponding papers in SWJ have between 3 and 5 stars for their vocabulary, with the overwhelming majority having 4 stars. If we accept the categories as a quality measure, then the datasets score high, but most do not score the top 5 stars. But we can at this stage only speculate as to the reasons for this. Due to the journal's depublishing strategy, we cannot easily track whether rejected dataset description papers were less than 4 stars on average.

We note that obtaining the fifth star is in fact not quite easy, because it actually requires third parties to acknowledge the independent value of the used vocabulary by establishing links. This means that the authors of a dataset cannot always actively improve their dataset in order to obtain the fifth star. The fifth star thus constitutes an indirect quality measure, by understanding a reuse by third parties as a type of endorsement of the vocabulary. Authors of a dataset can actively only obtain the fifth star by basing their dataset on a vocabulary that has already been established and is already linked to by others. But of course such a vocabulary may not exist yet in the topic area of the linked dataset being constructed. Note, that a 4 star dataset may become a 5 star dataset as time progresses and the utilized vocabulary is gaining impact.

Regarding the overall good quality of the vocabularies with respect to our rating, we of course acknowledge that the corresponding papers (and thus the underlying datasets) have undergone a rigorous review for the journal, and although a rating regarding our 5-star categories was not part of the review process, some of the categories - stars one through four - are arguably rather natural and would often be adhered to by authors concerned about a certain minimum quality of the artifact they create. And correspondingly, reviewers could be expected to look into such aspects.
This of course raises the open question where other datasets, i.e., datasets which do not have corresponding dataset description papers in the Semantic Web journal, fall with respect to the 5-star rating. If the profiles look very similar to the ratings described herein, i.e., they would mostly be four stars, then perhaps this could be taken as an argument that the 5-star rating is actually not very helpful in the sense that it is an already established practice. If the profiles look different, in particular if they include a significant body of ratings with fewer stars, then the hypothesis arises that the categories may indeed correlate with aspects of quality, although research will have to advance on the Linked Data quality front before this hypothesis can be investigated effectively.

More importantly, however, the fact that accepted dataset description papers typically clock in at 4 stars means that vocabularies and their quality are indeed considered important aspects of proper Linked Data publishing and that it may indeed be best practice to select vocabularies wisely and following certain criteria. The star rating proposed back in 2014 reminds us that in earlier years many Linked Data enthusiasts questioned the need for shared (and formal) vocabularies altogether and that this sentiment seems to be changing. We believe that proper vocabularies are a key driver for dataset discovery and reuse. Interestingly, the reuse of vocabularies themselves and best strategies for doing so (e.g., direct usage versus alignment) remains an open issue.

\section{References}

[1] R. Albertoni, M.D. Martino, S.D. Franco, V.D. Santis and P. Plini, EARTh: An environmental application reference thesaurus in the Linked Open Data cloud, Semantic Web 5(2) (2014), 165-171. doi:10.3233/SW-130122.

[2] S. Anibaldi, Y. Jaques, F. Celli, A. Stellato and J. Keizer, Migrating bibliographic datasets to the Semantic Web: The AGRIS case, Semantic Web 6(2) (2015), 113-120. doi:10. 3233/SW-130128.

[3] G.A. Atemezing, Ò. Corcho, D. Garijo, J. Mora, M. PovedaVillalón, P. Rozas, D. Vila-Suero and B. Villazón-Terrazas, Transforming meteorological data into linked data, Semantic Web 4(3) (2013), 285-290. doi:10.3233/SW-120089.

[4] K. Baierer, E. Dröge, K. Eckert, D. Goldfarb, J. Iwanowa, C. Morbidoni and D. Ritze, DM2E: A linked data source of digitised manuscripts for the digital humanities, Semantic Web 8(5) (2017), 733-745. doi:10.3233/SW-160234.

[5] T. Berners-Lee, Linked data, 2006, https://www.w3.org/ DesignIssues/LinkedData.html.

[6] E.D. Buccio, G.M.D. Nunzio and G. Silvello, A curated and evolving linguistic linked dataset, Semantic Web 4(3) (2013), 265-270. doi:10.3233/SW-2012-0083. 
[7] G. Candela, P. Escobar, R.C. Carrasco and M. Marco-Such, Migration of a library catalogue into RDA linked open data, Semantic Web (2018), To appear.

[8] S. Capadisli, S. Auer and A.-C.N. Ngomo, Linked SDMX data, Semantic Web 6(2) (2015), 105-112. doi:10.3233/SW-130123.

[9] C. Caracciolo, A. Stellato, A. Morshed, G. Johannsen, S. Rajbhandari, Y. Jaques and J. Keizer, The AGROVOC linked dataset, Semantic Web 4(3) (2013), 341-348. doi:10.3233/SW130106

[10] I. Celino, Geospatial dataset curation through a location-based game, Semantic Web 6(2) (2015), 121-130. doi:10.3233/SW130129

[11] C. Chichester, O. Karch, P. Gaudet, L. Lane, B. Mons and A. Bairoch, Converting neXtProt into linked data and nanopublications, Semantic Web 6(2) (2015), 147-153. doi:10.3233/ SW-140149.

[12] G. Correndo and N. Shadbolt, Linked data representation of the nomenclature of territorial units for statistics, Semantic Web 4(3) (2013), 251-256.

[13] E. Daga, M. d'Aquin, A. Adamou and S. Brown, The Open University linked data - data.open.ac.uk, Semantic Web 7(2) (2016), 183-191. doi:10.3233/SW-150182.

[14] V. de Boer, J. Wielemaker, J. van Gent, M. Oosterbroek, M. Hildebrand, A. Isaac, J. van Ossenbruggen and G. Schreiber, Amsterdam Museum linked open data, Semantic Web 4(3) (2013), 237-243. doi:10.3233/SW-2012-0074.

[15] G. de Melo, Lexvo.org: Language-related information for the linguistic linked data cloud, Semantic Web 6(4) (2015), 393400. doi:10.3233/SW-150171.

[16] S. Dietze, D. Taibi and M. d'Aquin, Facilitating scientometrics in learning analytics and educational data mining - The LAK dataset, Semantic Web 8(3) (2017), 395-403. doi:10.3233/SW150201.

[17] C. Dijkshoorn, L. Jongma, L. Aroyo, J. van Ossenbruggen, G. Schreiber, W. ter Weele and J. Wielemaker, The Rijksmuseum collection as linked data, Semantic Web 9(2) (2018), In this issue.

[18] M. Dojchinovski and T. Vitvar, Linked web APIs dataset, Semantic Web (2018), To appear.

[19] J. Eckle-Kohler, J.P. McCrae and C. Chiarcos, LemonUby A large, interlinked, syntactically-rich lexical resource for ontologies, Semantic Web 6(4) (2015), 371-378. doi:10.3233/ SW-140159.

[20] M. Ehrmann, G. Jacquet and R. Steinberger, JRC-Names: Multilingual entity name variants and titles as linked data, Semantic Web 8(2) (2017), 283-295. doi:10.3233/SW-160228.

[21] M. Färber, C. Menne and A. Harth, A linked data wrapper for CrunchBase, Semantic Web (2018), To appear.

[22] J. Gracia, M. Villegas, A. Gómez-Pérez and N. Bel, The apertium bilingual dictionaries on the web of data, Semantic Web 9(2) (2018), In this issue.

[23] R.D. Gratta, F. Frontini, F. Khan and M. Monachini, Converting the PAROLE SIMPLE CLIPS lexicon into RDF with lemon, Semantic Web 6(4) (2015), 387-392. doi:10.3233/SW140168.

[24] P. Hitzler and K. Janowicz, Linked data, big data, and the 4th paradigm, Semantic Web 4(3) (2013), 233-235. doi:10.3233/ SW-130117.

[25] K. Höffner, M. Martin and J. Lehmann, LinkedSpending: OpenSpending becomes linked open data, Semantic Web 7(1) (2016), 95-104. doi:10.3233/SW-150172.
[26] A. Hogan, P. Hitzler and K. Janowicz, Linked dataset description papers at the semantic web journal: A critical assessment, Semantic Web 7(2) (2016), 105-116. doi:10.3233/SW-160216.

[27] A. Isaac and B. Haslhofer, Europeana linked open data - data.europeana.eu, Semantic Web 4(3) (2013), 291-297. doi:10.3233/SW-120092.

[28] A. Ismayilov, D. Kontokostas, S. Auer, J. Lehmann and S. Hellmann, Wikidata through the eyes of DBpedia, Semantic Web (2018), To appear.

[29] K. Janowicz, P. Hitzler, B. Adams, D. Kolas and C. Vardeman, Five stars of linked data vocabulary use, Semantic Web 5(3) (2014), 173-176. doi:10.3233/SW-140135.

[30] T. Kauppinen, G.M. de Espindola, J. Jones, A. Sànchez, B. Gräler and T. Bartoschek, Linked Brazilian Amazon rainforest data, Semantic Web 5(2) (2014), 151-155. doi:10.3233/ SW-130113.

[31] H. Khrouf and R. Troncy, EventMedia: A LOD dataset of events illustrated with media, Semantic Web 7(2) (2016), 193199. doi:10.3233/SW-150184.

[32] L. Lefort, A. Haller, K. Taylor, G. Squire, P. Taylor, D. Percival and A. Woolf, The ACORN-SAT linked climate dataset, Semantic Web 8(6) (2017), 959-967. doi:10.3233/SW-160241.

[33] E. Mäkelä, K. Hypén and E. Hyvönen, Fiction literature as linked open data - The BookSampo dataset, Semantic Web 4(3) (2013), 299-306. doi:10.3233/SW-120093.

[34] M. Martin, C. Stadler, P. Frischmuth and J. Lehmann, Increasing the financial transparency of European Commission project funding, Semantic Web 5(2) (2014), 157-164. doi:10.3233/ SW-130116.

[35] J.A.M. narro Giménez, M.E. na Aranguren, B. VillazónTerrazas and J.T. Fernández-Breis, Translational research combining orthologous genes and human diseases with the OGOLOD dataset, Semantic Web 5(2) (2014), 145-149. doi:10.3233/SW-130109.

[36] A.M. no Peñuela, A. Ashkpour, C. Guéret and S. Schlobach, CEDAR: The Dutch historical censuses as linked open data, Semantic Web 8(2) (2017), 297-310. doi:10.3233/SW-160233.

[37] N. Queralt-Rosinach, T. Kuhn, C. Chichester, M. Dumontier, F. Sanz and L.I. Furlong, Publishing DisGeNET as nanopublications, Semantic Web 7(5) (2016), 519-528. doi:10.3233/SW150189.

[38] L. Rietveld, W. Beek, R. Hoekstra and S. Schlobach, Metadata for a lot of LOD, Semantic Web 8(6) (2017), 1067-1080. doi:10.3233/SW-170256.

[39] V. Rodriguez-Doncel, A.A. Krisnadhi, P. Hitzler, M. Cheatham, N. Karima and R. Amini, Pattern-based linked data publication: The linked chess dataset case, in: Proceedings of the 6th International Workshop on Consuming Linked Data Co-Located with 14th International Semantic Web Conference (ISWC 2105), Bethlehem, Pennsylvania, US, October 12th, 2015, O. Hartig, J. Sequeda and A. Hogan, eds, CEUR Workshop Proceedings, Vol. 1426, 2015.

[40] M. Sabou, I. Arsal and A.M.P. Braşoveanu, TourMISLOD: A tourism linked data set, Semantic Web 4(3) (2013), 271-276. doi:10.3233/SW-2012-0087.

[41] M. Salvadores, P.R. Alexander, M.A. Musen and N.F. Noy, Bioportal as a dataset of linked biomedical ontologies and terminologies in RDF, Semantic Web 4(3) (2013), 277-284. doi:10.3233/SW-2012-0086. 
[42] G. Sérasset, DBnary: Wiktionary as a lemon-based multilingual lexical resource in RDF, Semantic Web 6(4) (2015), 355361. doi:10.3233/SW-140147.

[43] M.A. Sherif and A.-C.N. Ngomo, Semantic Quran, Semantic Web 6(4) (2015), 339-345. doi:10.3233/SW-140137.

[44] N. Simou, J.-P. Evain, N. Drosopoulos and V. Tzouvaras, Linked European television heritage, Semantic Web 4(3) (2013), 323-329. doi:10.3233/SW-130104.

[45] M. Solanki, J. Skarka and C. Chapman, Linked data for potential algal biomass production, Semantic Web 4(3) (2013), 331340. doi:10.3233/SW-130105.

[46] C. Torniai, D. Bourges-Waldegg and S. Hoffmann, eaglei: Biomedical research resource datasets, Semantic Web 6(2) (2015), 139-146. doi:10.3233/SW-130133.

[47] M. Vafopoulos, M. Meimaris, I. Anagnostopoulos, A. Papantoniou, I. Xidias, G. Alexiou, G. Vafeiadis, M. Klonaras and V. Loumos, Public spending as LOD: The case of Greece, Semantic Web 6(2) (2015), 155-164. doi:10.3233/SW-140155.

[48] A. van Aggelen, L. Hollink, M. Kemman, M. Kleppe and H. Beunders, The debates of the European Parliament as linked open data, Semantic Web 8(2) (2017), 271-281. doi:10.3233/ SW-160227.
[49] D. Vila-Suero, B. Villazón-Terrazas and A. Gómez-Pérez, datos.bne.es: A library linked data dataset, Semantic Web 4(3) (2013), 307-313. doi:10.3233/SW-120094.

[50] M. Villegas and N. Bel, PAROLE/SIMPLE 'Lemon' ontology and lexicons, Semantic Web 6(4) (2015), 363-369. doi:10. 3233/SW- 140148 .

[51] J. Weaver and P. Tarjan, Facebook linked data via the graph API, Semantic Web 4(3) (2013), 245-250. doi:10.3233/SW2012-0078.

[52] P. Westphal, C. Stadler and J. Pool, Countering language attrition with PanLex and the web of data, Semantic Web 6(4) (2015), 347-353. doi:10.3233/SW-140138.

[53] B. Zapilko, J. Schaible, P. Mayr and B. Mathiak, TheSoz: A SKOS representation of the thesaurus for the social sciences, Semantic Web 4(3) (2013), 257-263. doi:10.3233/SW-20120081.

[54] A. Zaveri, J. Lehmann, S. Auer, M.M. Hassan, M.A. Sherif and M. Martin, Publishing and interlinking the Global Health Observatory dataset, Semantic Web 4(3) (2013), 315-322. doi:10. 3233/SW-130102.

[55] A. Zaveri, A. Rula, A. Maurino, R. Pietrobon, J. Lehmann and S. Auer, Quality assessment for linked data: A survey, Semantic Web 7(1) (2016), 63-93. doi:10.3233/SW-150175. 